\title{
Inhaltsübersicht zu Teil 1 und Teil 2
}

Teil 1

1 Über das Messen

2 Vektoren

3 Eindimensionale Bewegung

4 Bewegung in einer Ebene

5 Dynamik I

6 Dynamik II

7 Arbeit und Energie

8 Erhaltung der Energie

9 Erhaltung des Impulses

10 Der Stoß

11 Die Drehbewegung

12 Drehmoment, Drehimpuls und Trägheitsmoment

13 Erhaltung des Drehimpulses

14 Gleichgewicht starrer Körper

15 Schwingungen

16 Gravitation

17 Statik der Flüssigkeiten und Gase

18 Dynamik der Flüssigkeiten und Gase

19 Wellen in elastischen Medien

20 Schallwellen

21 Temperatur

22 Wärme und der erste Hauptsatz der Thermodynamik

23 Kinetische Gastheorie I

24 Kinetische Gastheorie II

25 Entropie und der zweite Hauptsatz der Thermodynamik Ergänzungen Anhang

\section{Teil 2}

26 Ladung und Materie

27 Das elektrische Feld

28 Das Gaußsche Gesetz 
VIII Inhaltsübersicht zu Teil 1 und Teil 2

29 Das elektrische Potential

30 Kondensator und Dielektrikum

31 Strom und Widerstand

32 Spannungsquellen und Stromkreise

33 Das magnetische Feld

34 Das Magnetfeld elektrischer Ströme

35 Das Induktionsgesetz

36 Selbstinduktion und Gegeninduktion

37 Magnetische Eigenschaften der Materie

38 Elektromagnetische Schwingungen

39 Wechselströme

40 Die Maxwellschen Gleichungen

41 Elektromagnetische Wellen

42 Natur und Ausbreitungseigenschaften des Lichts

43 Reflexion und Brechung - Ebene Wellen und ebene Grenzflächen

44 Reflexion und Brechung - Kugelwellen und sphärische Grenzflächen

45 Interferenz

46 Beugung

47 Gitter und Spektren

48 Polarisation

49 Licht und Quantenphysik

50 Die Wellennatur der Materie

51 Struktur der Atome

52 Atomphysik: drei ausgewählte Themen

53 Elektrische Leitung in Festkörpern

54 Einführung in die Kernphysik

55 Kernenergie

56 Physik der Elementarteilchen und Kosmologie

Ergänzungen

Anhang 